\title{
Stability Analysis of Networked Control Systems with Multi-Packet Dropout based on Switched System Approach
}

\author{
Zijian Dong, Liang Tian, Huanhuan Luo, Guiping Zhou, Li Wang
}

\begin{abstract}
Networked control system models with packet dropout in multi-packet transmission were established under hypothetical conditions in this paper, and the system was seen as a switched system. The causes of packet dropout in networked control system are analyzed in view of single-packet transmission and multi-packet transmission respectively. Based on Lyapunov stability theory, the property of the networked control system with multi-packet dropout was analyzed from the point of view of an asynchronous dynamic system. The method which determined the multi-packet dropout boundary to keep the system steady was given. The simulation results show the influences of multi-packet dropout on the system performance and prove the validity of the analytical method proposed in this paper.
\end{abstract}

Keywords-networked control system, multi-packet dropout, switched system, asynchronous dynamic system, stability analysis.

\section{INTRODUCTION}

$\mathrm{N}$ etworked control system is a control system with controllers, actuators and sensors communicating through a communication network. Networked control system has attracted the attention of the scientific community in the last decades due to its many benefits such as lower weight and power requirements, high reliability and flexibility, ease of installation and maintenance, etc. Typical examples are traffic monitoring, large-scale distributed industrial processes, distributed software systems in cloud computing architectures, spacecraft, fieldbus systems, intelligent traffic systems, autonomous robots, multi-agent systems and satellite clusters, etc. Whereas, there are still many challenges. The insertion of communication network in feedback control loop complicates the analysis and design of networked control system because many theorems and assumptions in traditional control theory cannot be directly applied to networked control systems. When

This work was supported in part by the National Key R\&D Program of China No.2017YFB0902100.

Zijian Dong is with the Department of Automation, North China Electric Power University, Baoding 071000, Hebei, China (corresponding author; e-mail: dongzijian268@foxmail.com).

Liang Tian is with the Department of Automation, North China Electric Power University, Baoding 071000, Hebei, China.

Huanhuan Luo is with the State Grid Liaoning Electric Power Co., Ltd, Shenyang 110006, Liaoning, China.

Guiping Zhou is with the Electric Power Research Institute of State Grid Liaoning Electric Power Co., Ltd, Shenyang 110006, Liaoning, China.

$\mathrm{Li}$ Wang is with the Electric Power Research Institute of State Grid Liaoning Electric Power Co., Ltd, Shenyang 110006, Liaoning, China. the controllers, actuators and sensors use the network to transmit data and control information, packet dropout, including one packet and multi-packet, will inevitably occur as the impact of network communication in the networked control system.

Generally speaking, a steady network control system can only withstand a certain proportion of data loss, when the packet dropout rate exceeds a certain value, the system will be unstable. So far, in the existing works, there have been several papers which addressed the stability analysis, control problems for networked control system. The literature [1] proposed a suitable networked control scheme and its stability analysis framework have been developed for controlling inherent electromechanical oscillatory dynamics observed in power systems. A novel adaptive two-level quantizer, which generates 1-b per sample and can be used for a class of industrial networked control systems in literature [2]. A novel interval delay system approach is used for stability analysis and control synthesis for networked control systems with two static quantizers[3]. Stability analysis and state feedback controller design of networked control systems with multiple-packet transmission and packet dropout in both the sensor-to-controller and controller-to-actuator are studied in [4, 5]. However, networked switched control systems, whose switched plant with all actuators and sensors, and switched controllers connected by communication channels, have not drawn attention in the multi-packet dropout until recently.

The main contributions of this paper are shown in the following aspects. First, the networked control system models with packet dropout in multi-packet transmission are established and the causes of packet dropout in networked control system are analyzed. Secondly, the system performance of networked control system with multi-packet dropout under different circumstances is analyzed from the point of view of asynchronous dynamic system and the method which determined the multi-packet loss boundary to keep the system stable is given. A theorem for exponential stability of closed-loop systems with data packet dropouts is proposed, and two simulation examples are given to verify the effectiveness of the proposed method above.

\section{SYSTEM MODELING}

It is necessary to model the multi-packet transmission as the control signal must be divided into multiple-packet for 
transmission when the data collected by the sensors is larger than the maximum transmission range.

First, make the following assumptions:

Assumption 1: The delay between the controllers and the sensors can be neglected, that is to say, when the data packet exceeds the maximum transmission delay, the data packet is assumed to be lost.

Assumption 2: The data packet loss is assumed to be a random process.

Assumption3: The controllers and actuator are event-driven and the sensors are time-driven.

Networked control system is studied when there are multiple-packet dropout between the sensors and controllers, and it is modeled as a switched system, and then the stability problem of networked control system can be transformed into that of switched system [6]. The stability of subsystems and random packet dropout are considered together since the stability of switched systems is not only related to subsystems, but also to the switched time [7].

The networked control system is modeled as a switched system with a certain probability switch. When the switch is at $S_{1}$, the data packet $y_{1}(k t)$ is transmitted to the controller. If the switch is at $S_{2}$, the data packet $y_{2}(k t)$ is transmitted to the controller. If the switch is at $S_{n}$, the data packet $y_{n}(k t)$ is transmitted to the controller.

The structure of networked control system with multi-packet dropout is shown as Fig.1.

Suppose that the process model is

$$
\left\{\begin{array}{c}
x((k+1) t)=A_{p} x(k t)+B_{p} u(k t)+\alpha(k) \\
y(k t)=C_{p} x(k t)+\beta(k)
\end{array},\right.
$$

and suppose that the state equation of the controller is

$$
\left\{\begin{array}{c}
\hat{x}((k+1) t)=A_{c} \hat{x}(k t)+B_{c} \hat{y}(k t) \\
u(k t)=C_{c} \hat{x}(k t)+D_{c} \hat{y}(k t)
\end{array},\right.
$$

where $x(k t) \in R^{n}, t$ is sampling period, $k \in n, A_{p}=e^{A t}$, $B_{p}=\int_{0}^{t} e^{A s} B d s, A \in R^{n \times n}, B \in R^{n \times 1}, C_{p} \in R^{1 \times n}, C_{c} \in R^{1 \times n}$, $\alpha(k)$ and $\beta(k)$ are noises.

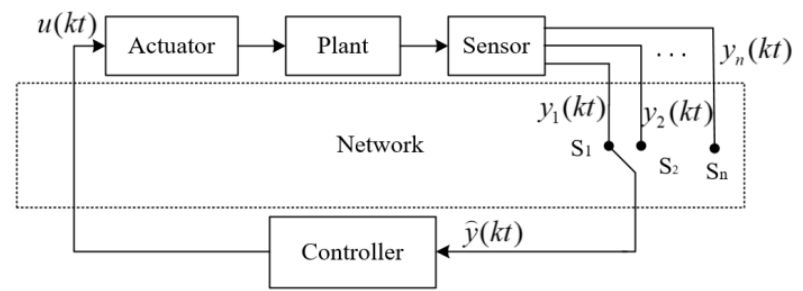

Fig.1. Networked control system structure with multi-packet dropout

The system outputs are divided into multiple data packets through multi-packet transmission in networked control system. Not considering the disorder of time series [8]. Suppose the data packets are sent in the following order by sensors: $S_{1}-S_{2}-\ldots-S_{n}-S_{1}-S_{2}-\ldots$, and when the switch is located at $S_{i}$, it means that the data packet $y_{i}(k t)$ is transmitted in the network. Let:

$$
y(k t)=\left[\begin{array}{c}
y_{1}(k t) \\
y_{2}(k t) \\
\ldots \\
y_{n}(k t)
\end{array}\right], \hat{y}(k t)=\left[\begin{array}{c}
\hat{y}_{1}(k t) \\
\hat{y}_{2}(k t) \\
\ldots \\
\hat{y}_{n}(k t)
\end{array}\right],
$$

when the switch is located at $S_{1}$, yielding

$$
\hat{y}_{2}(k t)=y_{2}((k-1) t), \ldots, \hat{y}_{n}(k t)=y_{n}((k-1) t),
$$

when the switch is located at $S_{2}$, yielding

$$
\begin{gathered}
\hat{y}_{1}(k t)=y_{1}((k-1) t), \hat{y}_{2}(k t)=y_{2}(k t), \ldots, \\
\hat{y}_{n}(k t)=y_{n}((k-1) t),
\end{gathered}
$$

when the switch is located at $S_{n}$, yielding

$$
\begin{gathered}
\hat{y}_{1}(k t)=y_{1}((k-1) t), \hat{y}_{2}(k t)=y_{2}((k-1) t), \ldots, \\
\hat{y}_{n}(k t)=y_{n}(k t),
\end{gathered}
$$

when the data packets transmitted in the network are $y_{i}(k t)$, let the augmented vector be

$$
z(k t)=\left[x^{T}(k t), \hat{x}^{T}(k t), \hat{y}^{T}(k t)\right]^{T} .
$$

when the switch is located at $S_{1}$, the state equation of the process is

$$
x((k+1) t)=A_{p} x(k t)+B_{p} C_{c} \hat{x}(k t)+B_{p} D_{c} \hat{y}^{T}(k t)+\alpha(k) .
$$

The state equation of the controller is

$$
\begin{gathered}
\hat{x}((k+1) t)=A_{c} \hat{x}(k t)+B_{c} \hat{y}(k t), \\
\hat{y}_{1}(k t)=y_{1}(k t), \hat{y}_{2}(k t)=y_{2}((k-1) t), \ldots, \\
\hat{y}_{n}(k t)=y_{n}((k-1) t) .
\end{gathered}
$$

Since no data packet loss occurred, there are

$$
\begin{gathered}
\hat{y}_{1}(k t)=y_{1}(k t), \\
\hat{y}_{2}(k t)=y_{2}((k-1) t)=\hat{y}_{2}((k-1) t), \\
\ldots \ldots \ldots \ldots \ldots \ldots, \ldots \ldots \ldots, \\
\hat{y}_{n}(k t)=y_{n}((k-1) t)=\hat{y}_{n}((k-1) t) .
\end{gathered}
$$

As above, the model of networked control system without multi-packet dropout is

$$
z((k+1) t)=A_{z} z(k t)+B_{z} \sigma(k),
$$




$$
\begin{gathered}
A_{z}=\left[\begin{array}{ccc}
A_{p} & B_{p} C_{p} & B_{p} D_{c} \\
0 & A_{c} & B_{c} \\
P_{0} & P_{1} & P_{2}
\end{array}\right], B_{z}=\left[\begin{array}{cc}
I & I \\
0 & 0 \\
C_{0} & I_{0}
\end{array}\right], \\
\sigma(k)=\left[\begin{array}{c}
\alpha(k) \\
\beta(k)
\end{array}\right],
\end{gathered}
$$

where the first row block of the blocking matrix $\left[\begin{array}{lll}P_{0} & P_{1} & P_{2}\end{array}\right]$ is the first row block of the blocking matrix $\left[C_{p} A_{p} \quad C_{p} B_{p} C_{c} \quad C_{p} B_{p} D_{c}\right]$, the lower right corner of matrix $P_{2}$ is a unity matrix, the others are 0 , the first row and the first column of the blocking matrix $C_{0}$ is the first row and the first column of the blocking matrix $C_{p}$, the others are 0 , the first row and the first column of the blocking matrix $I_{0}$ is a unity matrix, the others are 0 .

Assume $y_{i}(k t)$ is now transmitted in the network, but it is lost, one has $\hat{y}(k t)=\hat{y}((k-1) t)$. Let the augmented vector be

$$
z(k t)=\left[\begin{array}{lll}
x^{T}(k t) & \hat{x}^{T}(k t) & \hat{y}^{T}(k t)
\end{array}\right]^{T},
$$

and the process state equation is

$$
x((k+1) t)=A_{p} x(k t)+B_{p} C_{c} \hat{x}(k t)+B_{p} D_{c} \hat{y}^{T}(k t)+\alpha(k),
$$

and the state equation of the controller is

$$
\hat{x}((k+1) t)=A_{c} \hat{x}(k t)+B_{c} \hat{y}(k t) .
$$

Then model of networked control system with multi-packet dropout can be written as below:

$$
\begin{aligned}
& z((k+1) t)=A_{1} z(k t)+\alpha(k), \\
& A_{1}=\left[\begin{array}{ccc}
A_{p} & B_{p} C_{c} & B_{p} D_{c} \\
0 & A_{c} & B_{c} \\
0 & 0 & I
\end{array}\right] .
\end{aligned}
$$

\section{StabiLity ANALYSIS}

When network transmission is introduced into the control system, it will inevitably lead to the data packet dropout besides the effect of time delay. There are three main reasons.

First, as the core of network nodes, microprocessors cannot always ensure that they can work properly, and occasional failures are inevitable. When a node fails, it is obvious that the communication task will fail which will result in data packet dropout.

Second, conflicts are essentially inevitable in random access networks. In post-conflict situations, although most network communication protocols can realize the mechanism of conflict-relay, but the information retransmissions have set a timeout function, once the timeout arrives, the communication task will be abandoned, then data packet dropout occurs.

Third, in the actual system, the external environment will inevitably affect the quality of data transmission through the channel. Thousand disturbances cause physical signal dislocation, which cause the actual data distortion after it reaches the destination node. The perfect communication protocols have effective error detection or correction mechanism, and the error packets are usually discarded by the destination node. Similarly, although many communication protocols have a failure-retransmit mechanism, time-out may occur. Once the timeout arrives, the communication task will be abandoned which will still lead to data packet dropout.

Packet dropout often occurs after network transmission is introduced into the control system, besides the inevitable delay effect. A typical NCS structure with packet dropout is given in Fig.2[9].

The state vector of the process is denoted by $x$, the control variable is denoted by $u$, the input of disturbance is denoted by $n$, the controlled variable is denoted by $n^{\prime}$, the output of sensor is denoted by $y$, the measurement output of the controller through the network is denoted by $\hat{y}$.

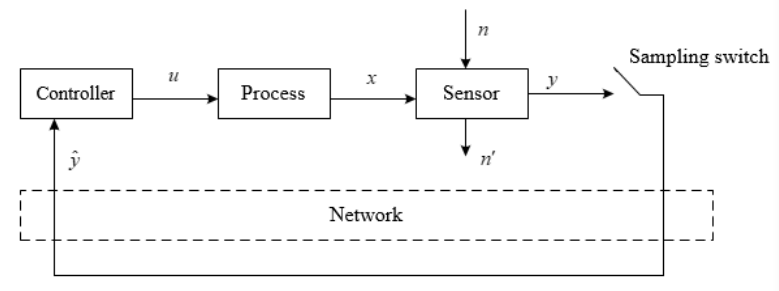

Fig.2. System block diagram with packet dropout

It indicates that the network works normally and no data packets are lost when the sampling switch is closed, whereas there is a loss of data packets when the sampling switch is disconnected. Sampled-data feedback control system allows partial data loss generally, but excessive packet loss may cause system unstable.

Consider the multi-packet dropout problem, a networked control system is actually an asynchronous dynamic system (ADS) which means that it is a system that includes both discrete and continuous dynamics. There are some conclusions about the stability of asynchronous dynamic systems below [10].

Lemma 1 : Consider an asynchronous dynamic system

$$
x(k+1)=f_{s}(x(k)), s=1,2, \ldots, N,
$$

if there is a Lyapunov function $V(x(k)): R^{n} \rightarrow R_{+}$and scalars $\alpha_{1}, \alpha_{2}, \ldots \alpha_{N}$, satisfy

$$
\begin{gathered}
\alpha_{1}^{r_{1}} \alpha_{2}^{r_{2}} \ldots \alpha_{N}^{r_{N}}>\alpha>1, \\
V\left(x(k+1)-V(x(k)) \leq\left(\alpha_{s}^{-2}-1\right) V(x(k)), s=1,2, \ldots, N,\right.
\end{gathered}
$$

then it can be said that the asynchronous dynamic system is exponentially stable. 
Lemma 2 : If the system is a difference equation $x((k+1) h)=\Phi_{s} x(k h)$, for each $s=1,2, \ldots N$, can find a corresponding Lyapunov function: $V(x(k h))=x^{T}(k h) P x(k h)$, and scalars $\alpha_{1}^{r_{1}} \alpha_{2}^{r_{2}} \ldots \alpha_{N}^{r_{N}}>\alpha>1$, then it can be said that the sufficient condition for exponential stability of the system is

$$
\begin{gathered}
r_{1} \log _{a} \alpha_{1}+r_{2} \log _{a} \alpha_{2}+\ldots+r_{N} \log _{a} \alpha_{N}>0, \\
\Phi_{s}^{T} P \Phi_{s} \leq \alpha_{s}^{-2} P,
\end{gathered}
$$

where $s=1,2, \ldots, N, a>1$

When a single packet is transmitted, the two states of a switch are used to describe the packet dropout and non-loss of packet, so the two states are two different events. Make the probability of packet dropout event to be $r$, then the successfully transmitted probability of data packet is $1-r$.

It can be seen from the above that the networked control system model with packet dropout is

$$
\begin{gathered}
z((k+1) h)=\Phi_{S_{i}} z(k h), \\
\Phi_{S_{1}}=\left[\begin{array}{cc}
\Phi & -\Gamma k \\
\Phi & -\Gamma k
\end{array}\right], \Phi_{S_{2}}=\left[\begin{array}{cc}
\Phi & -\Gamma k \\
0 & \mathrm{I}
\end{array}\right] .
\end{gathered}
$$

Theorem 1 : Let the Lyapunov function of the Switched system $z((k+1) h)=\Phi_{S_{i}} z(k h)$ is $V(x(k h))=x^{T}(k h) P x(k h)$, where $P$ is a symmetric positive definite matrix, assume the successful transmission rate of networked control systems is $r$, packet dropout rate is $1-r$, when there exist positive and constant numbers $a_{1}, a_{2}$, satisfy

$$
\begin{gathered}
a_{1}^{r} a_{2}^{1-r}>1 \\
{\left[\begin{array}{cc}
-P^{-1} & \alpha_{i} \Phi_{i} \\
\alpha_{i} \Phi_{i}^{\tau} & -P
\end{array}\right] \leq 0 i=1,2}
\end{gathered}
$$

then the networked control system is asymptotically stable.

The effective sampling period of the system is $h_{r}=h / r$ when the successful transmission rate of networked control system is $r$, where $h$ is the actual sampling period of the system. Namely, it is necessary to choose a larger sampling period when there are multi-packet dropouts through the system transmission in order to ensure the stability of the system. This theorem shows that when a higher sampling rate is adopted, the method of losing parts of the data packet can then be used to reduce the network load and to ensure the stability of the feedback system [11].

Assuming that the closed-loop system without data packet loss is stable, the following theorem exists for the system with data packet dropout.

Theorem 2 : Assuming that the closed-loop networked control system does not have data packet loss, the closed-loop system is stable, ( 1 ) if the open-loop system is stable, the closed-loop system is exponentially stable for transmission rate $0<r \leq 1$; ( 2 ) if the open-loop system is unstable, then for all $\frac{1}{1-\gamma_{1} / \gamma_{2}}<r \leq 1$, the closed-loop system is exponentially stable, where

$$
\begin{gathered}
r_{1}=\log _{a}\left[\lambda_{\max }^{2}(\Phi-\Gamma K)\right], r_{2}=\log _{a}\left[\lambda_{\max }^{2}(\Phi)\right] \\
\Phi=e^{A s}, \Gamma=\int_{0}^{T} e^{A s} B d s
\end{gathered}
$$

Proof: by the theorem 1, make $\beta_{i}=\alpha_{i}^{-2} i=1,2$, assume the data packet successful transmission rate $r$ satisfies

$$
\frac{\log _{a} \beta_{2}}{\log _{a} \beta_{2}-\log _{a} \beta_{1}}<r \leq 1, \beta<1,0<\beta_{1}<\beta_{2} .
$$

For the positive definite symmetric matrix $\mathrm{P}$, it has:

$$
\beta_{1} P \geq \Phi_{1}^{T} P \Phi_{1}, \beta_{2} P \geq \Phi_{2}^{T} P \Phi_{2},
$$

where $\Phi_{1}=\left[\begin{array}{cc}\Phi & -\Gamma \mathrm{K} \\ \Phi & -\Gamma \mathrm{K}\end{array}\right], \Phi_{2}=\left[\begin{array}{cc}\Phi & -\Gamma \mathrm{K} \\ 0 & \mathrm{I}\end{array}\right]$.

Then the networked control system is exponentially stable. The transmission rate $r$ depends on the choose of $\beta_{1}$ and $\beta_{2}$. Therefore, in order to make the networked control system progressively stable, there must be

$$
\beta_{1} \geq \lambda_{\max }^{2}\left(\Phi_{1}\right), \beta_{2} \geq \lambda_{\max }^{2}\left(\Phi_{2}\right) .
$$

If the lower bound of data packet successful transmission rate $r$ is to be obtained, $\log _{a} \beta_{2}-\log _{a} \beta_{1}$ must be minimized,

where

$$
\log _{a} \beta_{1}<0
$$

As

$$
\begin{gathered}
\lambda_{\max }^{2}\left(\Phi_{1}\right)=\lambda_{\max }^{2}(\Phi-\Gamma K), \lambda_{\max }^{2}\left(\Phi_{2}\right)=\left\{1, \lambda_{\max }^{2}(\Phi)\right\}, \\
\beta_{1} \geq \lambda_{\max }^{2}\left(\Phi_{1}\right), \beta_{2} \geq \lambda_{\max }^{2}\left(\Phi_{2}\right),
\end{gathered}
$$

so there is

$$
\beta_{1}=\lambda_{\max }^{2}(\Phi-\Gamma K), \beta_{2}=\left\{1, \lambda_{\max }^{2}(\Phi)\right\} .
$$

Therefore, if and only if the open-loop system $\Phi$ is stable, the networked control system is exponentially stable for any transmission rate $r$.

If the open-loop control system $\Phi$ is unstable, for

$$
\frac{1}{1-\gamma_{1} / \gamma_{2}}<r \leq 1
$$




$$
r_{1}=\log _{a}\left[\lambda_{\max }^{2}(\Phi-\Gamma K)\right], r_{2}=\log _{a}\left[\lambda_{\max }^{2}(\Phi)\right]
$$

and

$$
\frac{\log _{a} \beta_{2}}{\log _{a} \beta_{2}-\log _{a} \beta_{1}}<r \leq 1, \beta<1
$$

the closed control system is exponentially stable. This completes the proof.

\section{SimULATION}

\section{A. Simulation Example for Stable System}

Assume an open-loop stable discrete process

$$
\left\{\begin{array}{c}
x(k+1)=A x(k)+B u(k) \\
y(k)=C x(k)+D u(k)
\end{array}\right.
$$

where $A=\left[\begin{array}{cc}0.9 & 0.2 \\ 0 & 0.75\end{array}\right], B=\left[\begin{array}{l}0.004 \\ 0.022\end{array}\right], C=\left[\begin{array}{ll}6.3 & 0\end{array}\right], D=0$.

The open-loop block diagram of the system is shown in Fig.3.

It can be seen that the open-loop discrete control system is stable from the open-loop system output step response curve shown in Fig.4.

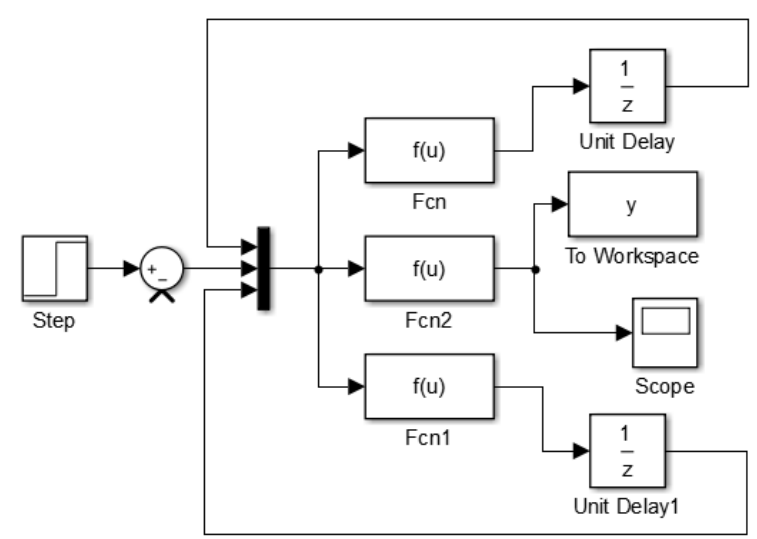

Fig.3.Open-loop block diagram

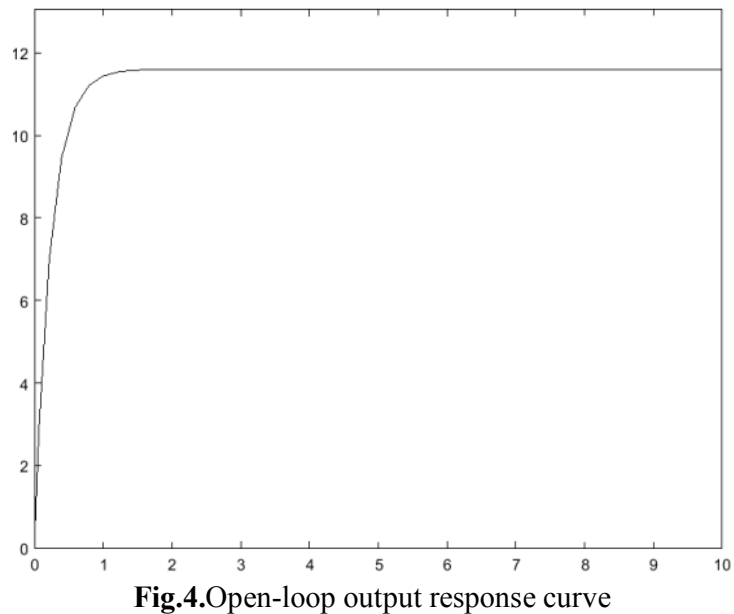

A state feedback block $u=K x$ is added in to the open-loop system shown in Fig.3, where $K=\left[\begin{array}{ll}5.4 & 7.8\end{array}\right]$. The simulation structure diagram of closed-loop control system without data packet dropout is shown in Fig.5.

It can be seen that the state feedback controller enables the system better steady-state performance from Fig.6. Assuming the packet dropout of the system is periodic, a packet loss segment is added into the closed-loop control system shown in Fig. 3, and the simulation structure of the networked control system with packet dropout is obtained and shown in Fig.7. The step response curve of the closed-loop networked control system with $10 \%$ packet dropout is given in Fig. 8 .

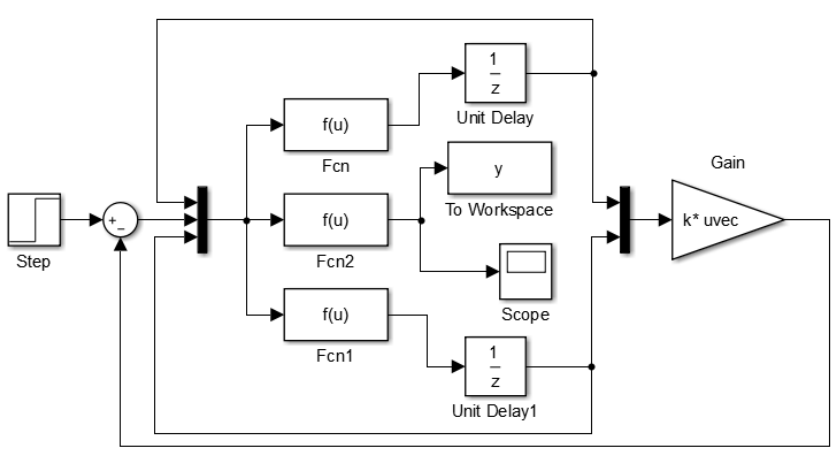

Fig.5.Closed-loop block diagram without packet loss 


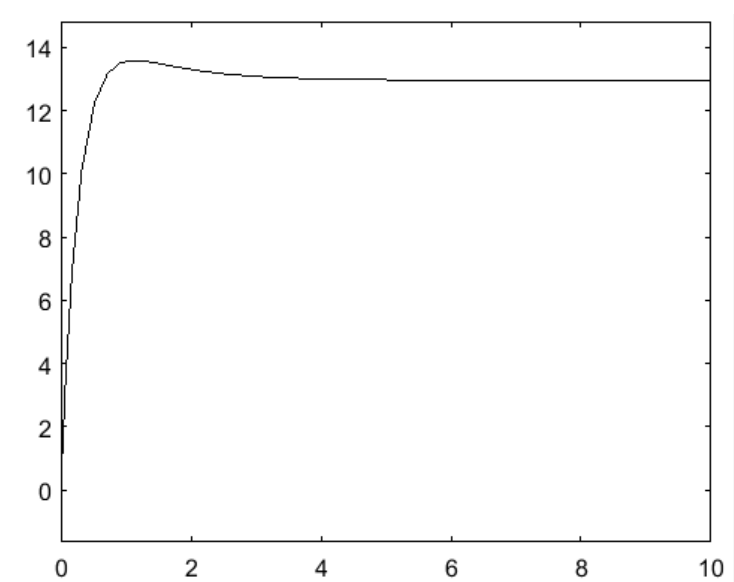

Fig.6.Open-loop output response curve without packet loss

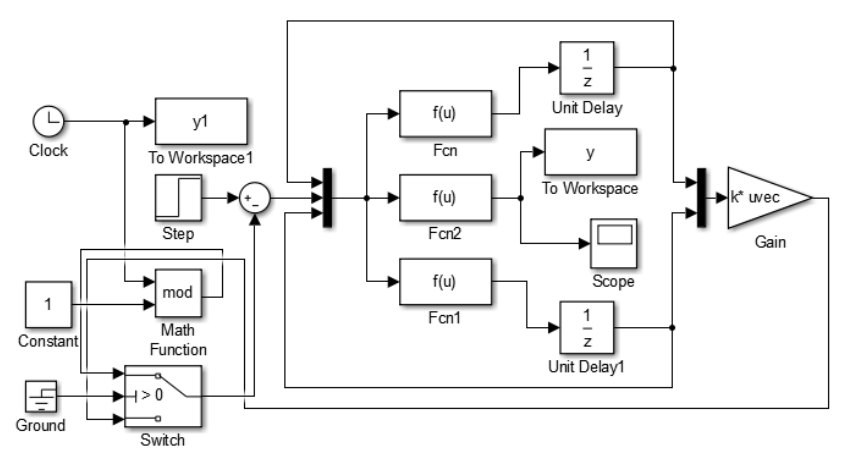

Fig.7.block diagram with packet loss

It can be seen that the output of the networked control system oscillates when packet dropout occurs, but the system remains stable from Fig.8. For open-loop stable systems, consider Fig.4 and Fig.6, packet dropout will have a certain impact on the stability of closed-loop systems, but it will not make the system unsteady.

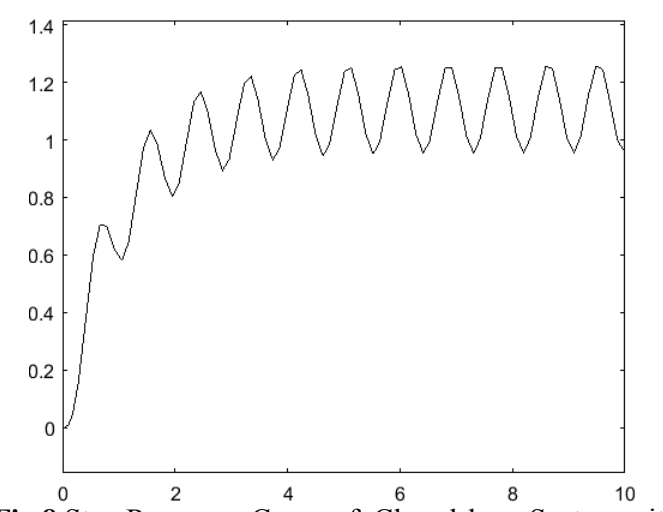

Fig.8.Step Response Curve of Closed-loop System with 10\% Packet Loss

\section{B. Simulation example for unstable system}

Assume a open-loop instability discrete control process:

$$
\left\{\begin{array}{c}
x(k+1)=A x(k)+B u(k) \\
y(k)=C x(k)+D u(k)
\end{array}\right.
$$

where $A=\left[\begin{array}{cc}1.2 & 0.1 \\ 0.5 & -0.2\end{array}\right], B=\left[\begin{array}{l}1 \\ 0\end{array}\right], C=\left[\begin{array}{ll}8 & 0\end{array}\right], D=0$.

A feedback segment $u=K x$ is added into the open-loop unstable system, where $K=\left[\begin{array}{ll}2.9202 & 0.6232\end{array}\right]$. The response curve is shown in Fig.9. The output response curves of the closed-loop system with different packet dropout rates are shown in Fig.10-12.

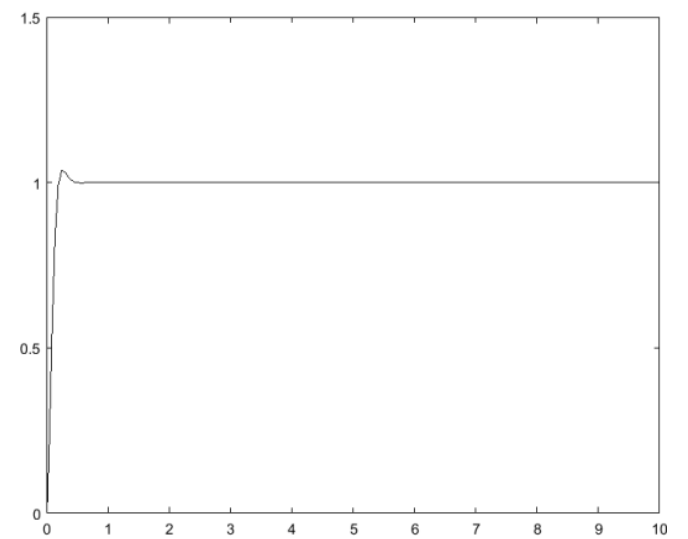

Fig.9. Response curve of closed-loop system with nopacket loss

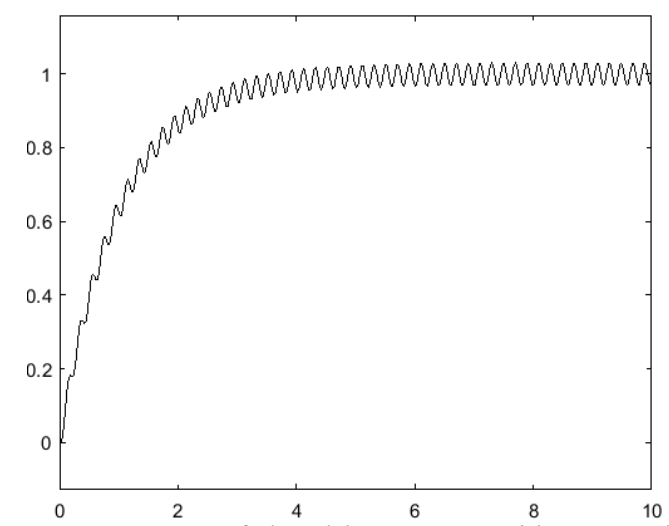

Fig.10.Response curve of closed-loop system with $10 \%$ packet loss

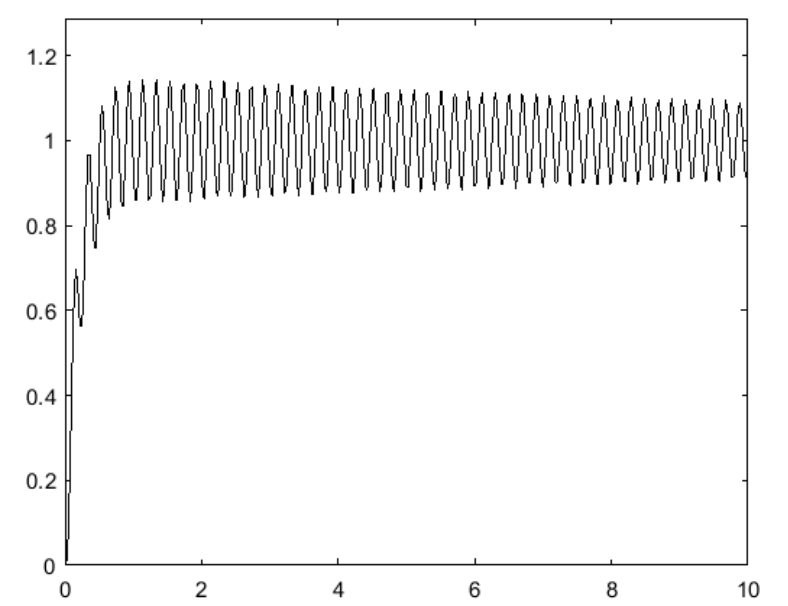

Fig.11. Response curve of closed-loop system with $20 \%$ packet loss

It can be seen from Fig10 and Fig.11 that when the packet loss rate is $10 \%$ and $20 \%$, the system stability is affected by the 
packet loss rate, but the system remains stable. While in Fig.12, when the packet loss rate increases to $30 \%$, the system becomes unstable. The simulation result shows that for open-loop unstable systems, when the packet loss rate exceeds a certain range, packet loss will not only affects the stability of the system, but also makes the system unstable.

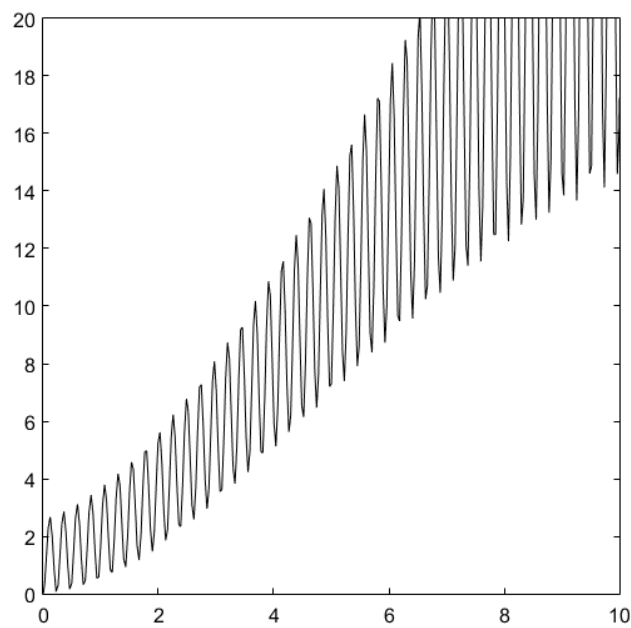

Fig.12. Response curve of closed-loop system with $30 \%$ packet loss

The control system and the feedback state matrix can get the packet dropout rate $r$ through the stability theorem, this parameter can be used to design the controller of the networked control system. The lower bound of the packet dropout rate $r$ must be got firstly when the matrix $A$ and $B$ are only known in order to design the state feedback matrix $K$, and then the controller can be designed through this parameter. Assum that there is no data packet dropout, and the gain matrix $K$ can make the closed-loop system stable when packets are transmission. The maximum packet loss boundary is defined as follows.

Assume the networked control system with data packet dropout in multi input-output state feedback as below.

$$
\left\{\begin{array}{c}
x(k+1)=\Phi x(k)+\Gamma_{1} u(k)+\Gamma_{2} w(k) \\
u(k)=-\mathrm{K} \lambda(k) x(k)
\end{array} .\right.
$$

For any given arbitrary packet loss rate $r<r_{\max }$, this system is mean square stable, $r_{\max }$ is referred to as the maximum packet loss boundary.

Define a matrix $\mathrm{E}=r \Phi \otimes \Phi+(1-r)(\Phi-\Gamma \mathrm{K}) \otimes(\Phi-\Gamma \mathrm{K})$, let $\mathrm{M}=r \Phi \otimes \Phi, \mathrm{N}=(1-r)\left(\Phi-\Gamma_{1} \mathrm{~K}\right) \otimes\left(\Phi-\Gamma_{1} \mathrm{~K}\right), \mathrm{M}$ is considered a perturbation of $\mathrm{N}, \mathrm{M}$ is equal to $\mathrm{N}$ when there is no packet dropout.

Define the stability domain:

$$
\sigma(S)=\inf \left\{\square \Delta \square_{2}: \Delta \in R^{n \times n}, S+\Delta \text { is unstable }\right\},
$$

where $\Delta$ is the unstable disturbance, $\square \Delta \square_{2}$ is a spectral norm. Assume $r(T-S)$ is the structural perturbation of $S$, and the regular system is $S+r(T-S)$, when there is no packet dropout, the regular system is $S$, when $r=1$, the regular system is $T$.Therefore, there are

$$
\square r_{\text {max }}(T-S) \square_{2} \geq \sigma(S),
$$

namely

$$
r_{\max } \geq \frac{\sigma(S)}{\square(T-S) \complement_{2}},
$$

and the stability radius $\sigma(S)$ needs to be determined.

Given a $n \times n$-dimensional stability matrix $\mathrm{A}$, and the stability radius $m_{\mathrm{c}}=\inf \square \mathrm{F} \square, \mathrm{F}$ is a $n \times n$-dimensional matrix, $\mathrm{A}+\mathrm{F}$ is an unstable matrix.

For the continuous system A and F, define

$$
m_{c}=\min _{w \in R} \xi_{n}(j w \mathrm{I}-A),
$$

where $\xi_{n}$ is a minimum singular value, I is an unity matrix.

Define the maximum attenuation rate of the system as below

$$
\alpha=-\max _{i} \operatorname{Re} \lambda_{i}(A),
$$

where $\lambda(A)$ is the characteristic value of $\mathrm{A}$. The lower bound of instability $\mathrm{A}+\mathrm{F}$ is that the minimum singular value of $\mathrm{A}$ is smaller than the maximum attenuation rate of $\mathrm{A}$.

The stability radius can be obtained by the following methods fr discrete systems.

Define

$$
\begin{gathered}
m_{d}=\min _{|z=1|} \xi_{n}(z \mathrm{I}-A), \\
\beta=1-r(A), \\
m_{d} \leq \beta,
\end{gathered}
$$

where $r(A)$ is the spectral radius of $\mathrm{A}, \beta$ is the attenuation rate of the discrete System.

Theorem 3 : For a given matrix $\mathrm{A}$, the equation

$$
\begin{gathered}
m_{d}=\min _{|z|=1} \xi_{n}(z \mathrm{I}-A), \\
\beta=1-r(A), \\
m_{d} \leq \beta,
\end{gathered}
$$

is valid if and only if

$$
\xi_{n}(z \mathrm{I}-A)=\min _{i}\left|\lambda_{i}(z \mathrm{I}-A)\right| .
$$

Proof: Let

$$
\lambda_{i}(A)=a_{i}+b_{i}, z=\cos \theta+\sin \theta \cdot j, \theta \in[-\pi, \pi],
$$

yielding

$$
|\lambda(z \mathrm{I}-A)|=\left(1+a_{i}^{2}+b_{i}^{2}-2 y\right)^{1 / 2},
$$




$$
y=a_{i} \cos \theta+b_{i} \sin \theta
$$

so that

$$
-\left(a_{i}^{2}+b_{i}^{2}\right)^{1 / 2} \leq y \leq\left(a_{i}^{2}+b_{i}^{2}\right)^{1 / 2}
$$

and

$$
\min _{|z|=1}|\lambda(z \mathrm{I}-A)| \leq\left[1-\left(a_{i}+b_{i}\right)^{1 / 2}\right]
$$

take the minimum $i$ on both sides of the equation, then we have

$$
\min _{i} \min _{|z|=1}|\lambda(z \mathrm{I}-A)| \leq \min _{|z|=1} \min _{i}\left[1-\left(a_{i}+b_{i}\right)^{1 / 2}\right]=1-r(A),
$$

from the theorem condition above, yielding

$$
\xi_{n}(x) \leq \lambda_{i}(x),
$$

so that

$$
\xi_{n}(z \mathrm{I}-A) \leq \min _{i}\left|\lambda_{i}(z \mathrm{I}-A)\right|
$$

Take the minimum values of the two sides of the upper formula in the unit circle, yielding

$$
\min _{|z|=1} \xi_{n}(z \mathrm{I}-A) \leq \min _{|z|=1} \min _{i}\left|\lambda_{i}(z \mathrm{I}-A)\right| .
$$
Because $\min _{|z|=1} \min _{i}\left|\lambda_{i}(z \mathrm{I}-A)\right|=1-r(A)$, by synthesizing the

upper two expressions, one has

$$
\min _{|z|=1} \xi_{n}(z \mathrm{I}-A) \leq 1-r(A) .
$$

By definition above, there has

$$
m_{d}=\min _{|z=1|} \xi_{n}(z \mathrm{I}-A)
$$

namely

$$
m_{d} \leq 1-r(A) .
$$

This completes the proof.

\section{CONCLUSION}

Based on the hypothesis of networked control system, this paper analyses the networked control system model with packet dropout through multi-packet transmission, and studies the causes of packet dropout in networked control system. The system performance of networked control system with multi-packet loss under different circumstances is analyzed from the point of view of asynchronous dynamic system, and the influence of multi-packet dropout on system performance is illustrated by simulation. The stability conditions and stability boundaries of NCS with data packet loss are obtained.

\section{REFERENCES}

[1] Singh, Abhinav Kumar, R. Singh, and B. C. Pal. "Stability Analysis of Networked Control in Smart Grids", IEEE Transactions on Smart Grid, vol. 6 , no.1, pp.381-390, 2017.

[2] Almakhles, Dhafer, et al. "An Adaptive Two-Level Quantizer for Networked Control Systems", IEEE Transactions on Control Systems Technology, pp.99:1-8, 2017.

[3] Mahmoud, Magdi S. "Fuzzy networked control systems with communication constraints", Ima Journal of Mathematical Control \& Information, vol. 34, no.2, pp.543-564, 2018.

[4] Liu, Yi Cai, et al. "Stability analysis for networked control systems with bilateral random delay and packet dropout", Control \& Decision, vol. 32, no. 9 , pp.1565-1573, 2017.

[5] Su, Meng, C. Jie, and S. Jian. "Observer-based output feedback control of networked control systems with non-uniform sampling and time-varying delay", International Journal of Solids \& Structures, vol. 48, no. 2, pp.1-11, 2017.

[6] Wu, Ying, and Y. Wu. "Mode-dependent robust stability and stabilisation of uncertain networked control systems via an average dwell time switched approach", Iet Control Theory \& Applications, vol.11, no. 11, pp.1726-1735, 2017.

[7] Wen, Shixi, and G. Ge. "Minimum Data Rate for Exponential Stability of Networked Control Systems with Medium Access Constraints", International Journal of Control Automation \& Systems, vol.16, no. 9, pp. $1-9,2018$.

[8] Lian, Bosen, Q. Zhang, and J. Li. "Sliding mode control for non-linear networked control systems subject to packet disordering via prediction method", IET Control Theory \& Applications, vol. 11, no.17, pp.3079-3088, 2017.

[9] Yu, Mei, et al. "Quantized Output Feedback Control of Networked Control Systems with Packet Dropout", International Journal of Control, Automation and Systems,vol. 16, no.5, pp.2559-2568, 2018.

[10] Zheng, Jia Chun, et al. "A fusion algorithm of target dynamic information for asynchronous multi-sensors", Microsystem Technologies, vol.24, no.2, pp.1-11, 2017.

[11] Hu, Songlin, et al. "Stabilization of Neural-Network-Based Control Systems via Event-Triggered Control With Nonperiodic Sampled Data", IEEE Transactions on Neural Networks \& Learning Systems, vol.29, no.3, pp.573-585, 2018. 